\title{
Purtscher's-like retinopathy as a rare complication of acute alcoholic pancreatitis
}

\author{
Michalina M. Wieczorek, Miłosz Caban, Adam Fabisiak, Ewa Małecka-Panas \\ Department of Digestive Tract Diseases, Medical University of Lodz, Lodz, Poland
}

Gastroenterology Rev 2021; 16 (2): 170-173

DOI: https://doi.org/10.5114/pg.2021.106669

Address for correspondence: Michalina M. Wieczorek, Department of Digestive Tract Diseases, Medical University of Lodz, Lodz, Poland, e-mail: michalina.wieczorek19@gmail.com

Acute pancreatitis (AP) is one of the most frequent gastrointestinal causes of hospital admission [1, 2]. There are a lot of risk factors of AP, but $70-75 \%$ of all cases of AP result from alcohol abuse or cholelithiasis [3]. Severe abdominal pain, vomiting, and nausea are the main symptoms of AP [2, 4]. However, manifestation from other organs may occur in the course of this disease.

Purtscher's-like retinopathy is associated with non-traumatic causes [5]. Its occurrence has been reported in many disorders such as antibiotic anaphylaxis, myocardial infarction, connective tissue diseases, renal failure, childbirth, and bone marrow transplant [5-8]. Furthermore, Purtscher's-like retinopathy is rare with an incidence rate of less than 0.24 cases per million, and it is also a complication of AP [5]. So far, several such cases have been reported in the literature [5, 9-11]. It must be emphasized that Purtscher's-like retinopathy occurs primarily in acute alcoholic pancreatitis (AAP), and there are few case reports of this type of ocular disorder in acute non-alcoholic pancreatitis: in the context of a Klatskin tumour or an acute kidney injury $[12,13]$. Nevertheless, there is only 1 case report of this retinopathy in acute non-alcoholic pancreatitis without concomitant diseases [14]. This ocular disorder manifests by sudden visual loss and typical changes in fundus examination, which is caused by a vaso-occlusive microvasculopathy [5]. The pathophysiology of Purtscher's-like retinopathy remains unclear, which contributes to the lack of targeted therapy of this pathology condition.

We report a case of Purtscher's-like retinopathy concomitant with macular oedema in AAP. A 32-year-old man with a history of alcohol abuse was admitted to the Department of Digestive Tract Diseases in Norbert Barlicki Memorial University Hospital with vomiting and severe epigastric pain radiating to the back. Blood tests showed an elevated white blood cell count of
$13.9 \mathrm{G} / \mathrm{l}$ (reference range (RR): 4.0-10.0 G/l), haemoglobin of $17 \mathrm{~g} / \mathrm{dl}$ (RR: $13.5-18.0 \mathrm{~g} / \mathrm{dl}), \mathrm{C}$-reactive protein of $2.7 \mathrm{mg} / \mathrm{l}$ (RR: 0.0-5.0 mg/l), liver enzymes: aspartate aminotransferase of 152 units per litre (U/I) (RR: 0-40 U/l), alanine aminotransferase of $108 \mathrm{U} / \mathrm{l}(\mathrm{RR}: 0-40 \mathrm{U} / \mathrm{l})$, serum pancreas enzymes: lipase of $2060 \mathrm{U} / \mathrm{l}$ (RR: 0-67 U/I), amylase of $314 \mathrm{U} / \mathrm{l}(\mathrm{RR}: 22-80 \mathrm{U} / \mathrm{l})$, glucose of $113 \mathrm{mg} / \mathrm{dl}$ (RR: $70-99 \mathrm{mg} / \mathrm{dl}$ ), serum creatinine of $0.63 \mathrm{mg} / \mathrm{dl}$ (RR: $0.72-1.18 \mathrm{mg} / \mathrm{dl}$ ), and ethanol of $0.76 \%$ o (RR: $0.0-0.20 \%$ ). An abdominal ultrasound was negative for cholelithiasis or biliary duct dilatation. The AAP was diagnosed, and analgesic treatment with intensive fluid therapy was introduced. He had no evidence of organ failure or shock. On the day after admission, the patient developed sudden bilateral visual impairment. Specifically, he complained of "problems with near vision" and "blurred vision". An ocular examination demonstrated equal, round, and reactive pupils, as well as intact extraocular movements. The visual acuities of both eyes were 5/40 using Snellen's chart. The slit-lamp examination showed an unremarkable anterior segment. After adequate pupillary dilatation, fundus examination revealed the presence of peripapillary cotton-wool spots in both eyes (Figure 1). Moreover, optical coherence tomography (OCT) presented the retinal thickening in the macular area of both eyes, which revealed bilateral macular oedema (Figure 2). Based on these results, Purtscher's-like retinopathy with maculopathy in both eyes was diagnosed. Because of low visual acuity and advanced bilateral macular oedema, symptomatic treatment with nepafenac in eye drops (thrice a day), intravenous hydrocortisone (300 $\mathrm{mg}$ a day in 2 divided doses), and oral pentoxifylline (400 mg 3 times a day) was started. Two days later, the visual acuities of both eyes improved, and they were 8/40. On the fourth day of hospitalization the macular oedemas were significantly reduced (Figure 3 ). 

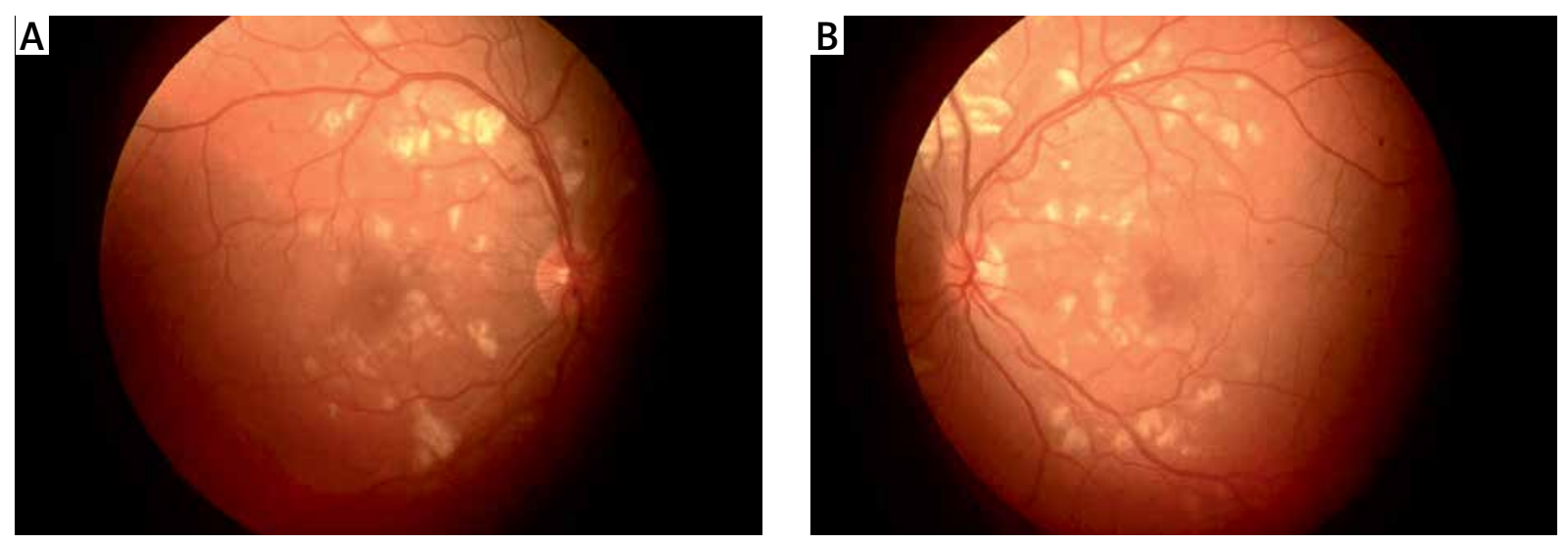

Figure 1. Multiple retinal cotton-wool spots in the funduscopic examination of both eyes
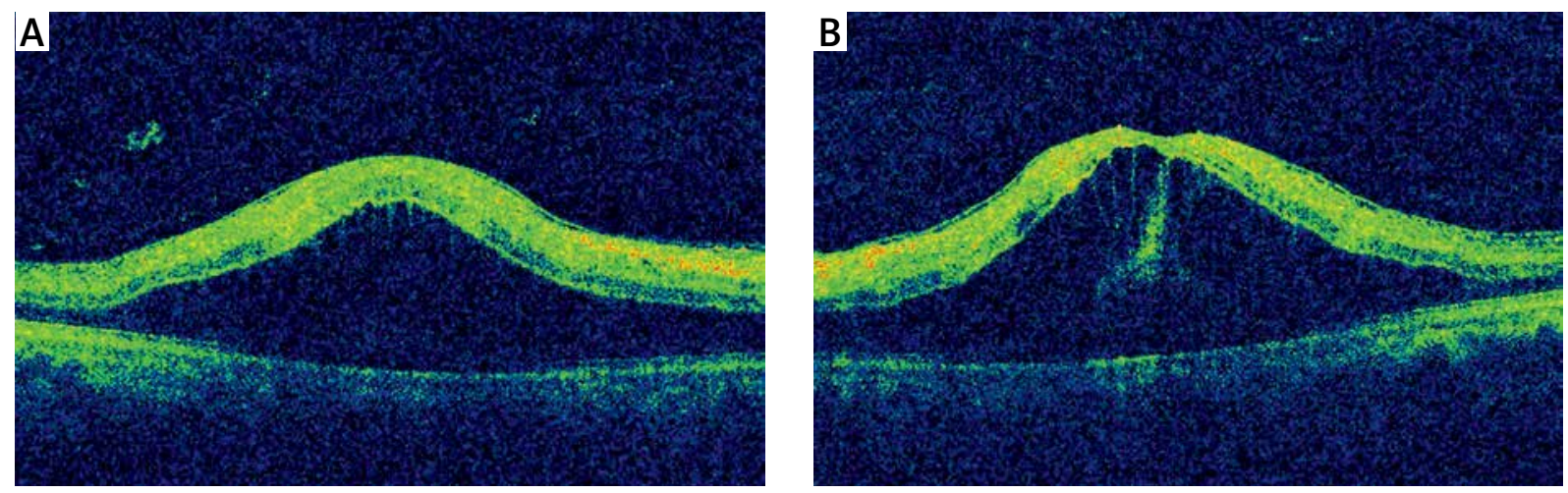

Figure 2. Optical coherence tomography showing macular oedema in both eyes at the start of treatment (A - right eye, B - left eye)


Figure 3. Optical coherence tomography showing reduced macular oedema in both eyes after 4 days of introduced therapy (A - right eye, B - left eye)

Ocular manifestation of AAP is unusual. Nevertheless, the incidence of sudden visual loss associated with changes in the retina (inter alia retinal haemorrhages and oedema) in AP was found to be $2 \%$ [9]. Purtscher's-like retinopathy represents one of the eye disorders in AAP [5, 9-11] and only rarely acute non-alcoholic pancreatitis [14]. The exact mechanisms that lead to its occurrence are still undiscovered. The caus- es of the development of Purtscher's-like retinopathy in AAP are unknown. Nevertheless, there are no data on alcohol-induced Purtscher's-like retinopathy without accompanying AP. On the other hand, alcohol abuse may contribute to the development of other retinal diseases among patients with diabetic retinopathy [15]. However, the most prevalent theory is that of retinal vessel occlusion by microemboli, which may originate 
from various kind of particles inter alia fat, fibrin clots, and leukocyte aggregates [12]. In the course of AP, the pancreatic damage contributes to exacerbated release of proteolytic enzymes into the circulation, which is responsible for the formation of C5a-induced leukocyte aggregate. As a consequence, retinal ischaemia occurs [12]. Furthermore, intraretinal haemorrhages, confluent cotton-wool spots, and Purtscher's flecken are specific changes detected in the posterior pole of the eye [5]. These changes cause blurred vision and the decrease of visual acuity. The criteria established by Agrawal and McKibbin are used to diagnose Purtscher's-like retinopathy $[9,16]$. They include the presence of multiple Purtscher's flecken and/or cotton-wool spots detected in funduscopic examination in one or both eyes, which occur in the course of an unassociated contributing illness $[9,16]$.

The lack of casual treatment is due to the unclear aetiology of Purtscher's-like retinopathy. However, there are several treatment options. Spontaneous visual recovery of at least 2 Snellen lines improvement, which would be equal to halving the visual angle, without treatment, occurs in $50 \%$ of cases [16]. From another side, high-dose, intravenous steroid treatment showed a good visual response within the first 2 weeks of treatment [17]. This effect results from the stabilization of damaged cellular membrane in eye neuronal tissue, the inhibition of formation of complement-activated leukocyte aggregation in vessels, and the suppression of oxygen-free radical production [5, 17]. Moreover, steroids have powerful anti-oedematous properties, which are used to reduce macular oedema [18]. Also, treatment with non-steroidal anti-inflammatory drugs (NSAIDs) seems to be effective in reducing oedema in patients with Purtscher's-like retinopathy [19]. Nepafenac, one of the NSAIDs, inhibits the cyclooxygenase enzymes responsible for prostaglandin production and is used to treat macular oedema [20]. In addition, Ortmaier et al. revealed that pentoxifylline may be the significant element of Purtscher's retinopathy therapy as a haemorheologic agent [21]. Our treatment with hydrocortisone, nepafenac, and pentoxifylline contributed to significant reduction of macular oedema and slightly improved visual acuity.

The prognosis of recovery in Purtscher's-like retinopathy is variable [5]. Nevertheless, further investigation of corticosteroids and also pentoxifylline use are necessary to determine if it can change the natural history of a patient with Purtscher's-like retinopathy. This case emphasizes the importance of exact medical history and examination, and raises the possibility of ophthalmological complications of AP. We recommend that ophthalmological consultation and ocular fundus examination are performed in patients with acute pancreatitis with blurred vision.

\section{Conflict of interest}

The authors declare no conflict of interest.

\section{References}

1. Yadav D, Lowenfels AB. The epidemiology of pancreatitis and pancreatic cancer. Gastroenterology 2013; 144: 1252-61.

2. Lankisch PG, Apte M, Banks PA. Acute pancreatitis. Lancet 2015; 386: 85-96.

3. Kamal A, Akshintala VS, Kamal MM, et al. Does etiology of pancreatitis matter? Differences in outcomes among patients with post-endoscopic retrograde cholangiopancreatography, acute biliary, and alcoholic pancreatitis. Pancreas 2019; 48: 574-8.

4. Bollen TL. Acute pancreatitis: international classification and nomenclature. Clin Radiol 2016; 71: 121-33.

5. Tariq T, Reaume M, Hammar D, et al. Blind after a binge: purtscher-like retinopathy in acute alcoholic pancreatitis. ACG Case Rep J 2019; 6: e00251.

6. Huang ZX, Zeng ZB, Xu ZP. Purtscher-like retinopathy associated with antibiotic anaphylaxis. Nan Fang Yi Ke Da Xue Xue Bao 2018; 38: 239-42.

7. Torrente-Nieto A, Gómez-Resa M, Castro-Guardiola A. Purtscher-like retinopathy and systemic lupus erythematosus. Med Clin 2018; 151: 504-5.

8. Sánchez Vicente JL, Castilla Martino M, Contreras Díaz M, et al. Purtscher-like retinopathy preceding acute renal failure. Arch Soc Esp Oftalmol 2018; 93: 198-201.

9. Subudhi P, Kanungo S, Subudhi NR. Purtscher's-like retinopathy in acute alcoholic pancreatitis. BMJ Case Rep 2016; 2016: bcr2016215485.

10. Ciortescu I, Cozma D, Epure B, et al. Purtscher's-like retinopathy: a rare complication of acute pancreatitis. J Gastrointest Liver Dis 2011; 20: 345.

11. Nema N, Ishrat S, Verma A, et al. Purtscher-like retinopathy in acute alcoholic pancreatitis. Oman J Ophthalmol 2016; 9: 110-2.

12. Massa R, Vale C, Macedo M, et al. Purtscher-like retinopathy. Case Rep Ophthalmol Med 2015; 2015: 421329.

13. Haque WMMU, Ananna MA, Haque HF, et al. Purtscher's retinopathy and renal cortical necrosis: two rare vaso-occlusive complications in a patient with acute pancreatitis: a case report. J Med Case Rep 2016; 10: 326.

14. Núñez L, Cubiella J, Moreno C, et al. Purtscher's retinopathy: a rare complication of acute non-alcoholic pancreatitis. Gastroenterol Hepatol 2003; 26: 541-4.

15. Wang S, Wang JJ, Wong TY. Alcohol and eye diseases. Surv Ophthalmol 2008; 53: 512-25.

16. Agrawal A, McKibbin M. Purtscher's retinopathy: epidemiology, clinical features and outcome. Br J Ophthalmol 2007; 91: 1456-9.

17. Wang AG, Yen MY, Liu JH. Pathogenesis and neuroprotective treatment in Purtscher's retinopathy. Jpn J Ophthalmol 1998; 42: 318-22. 
18. Fardeau C, Champion E, Massamba N, et al. Uveitic macular edema. Eye 2016; 30: 1277-92.

19. Nor-Masniwati S, Azhany Y, Zunaina E. Purtscher-like retinopathy following valsalva maneuver effect: case report. J Med Case Rep 2011; 5: 338.

20. Yüksel B, Karti Ö, Kusbeci T. Topical nepafenac for prevention of post-cataract surgery macular edema in diabetic patients: patient selection and perspectives. Clin Ophthalmol 2017; 11: 2183-90.

21. Ortmaier R, Resch H, Stieböck C, et al. Purtscher's retinopathy after intramedullary nailing of a femoral shaft fracture in a 20year old healthy female - report of a rare case and review of the literature. BMC Musculoskelet Disord 2014; 15: 42.

Received: 27.08 .2020

Accepted: 8.10.2020 